MATEC Web of Conferences 8, 03005 (2013)

DOI: $10.1051 /$ matecconf $/ 20130803005$

(C) Owned by the authors, published by EDP Sciences, 2013

\title{
Dynamical studies on the generation of periodic surface structures by femtosecond laser pulses
}

\author{
A. Rosenfeld ${ }^{1}$, S. Höhm ${ }^{1}$, J. Bonse ${ }^{2}$, J. Krüger ${ }^{2}$ \\ 1- Max-Born-Institut, Max-Born-Straße 2a, D-12489 Berlin, Germany \\ 2- BAM Bundesanstalt für Materialforschung und -prüfung, \\ Unter den Eichen 87, D-12205 Berlin, Germany \\ rosenfeld@mbi-berlin.de
}

\begin{abstract}
The dynamics of the formation of laser-induced periodic surface structures (LIPSS) on fused silica upon irradiation with linearly polarized fs-laser pulses (50 fs pulse duration, $800 \mathrm{~nm}$ center wavelength) is studied experimentally using a double pulse experiment with cross polarized pulse sequences and a transillumination femtosecond time-resolved $(0.1 \mathrm{ps}-1$ ns) pump-probe diffraction approach. The results in both experiments confirm the importance of the ultrafast energy deposition and the laser-induced free-electron plasma in the conduction band of the solids for the formation of LIPSS.
\end{abstract}

The formation of laser-induced periodic surface structures (LIPSS) upon irradiation of dielectrics $\left(\mathrm{SiO}_{2}\right)$ with multiple double-pulse irradiation sequences consisting of equal energy linearly polarized Ti:sapphire femtosecond laser pulse pairs (pulse duration $50 \mathrm{fs}$, central wavelength $\sim 800 \mathrm{~nm}$ ) is studied experimentally. The temporal delay between the individual cross-polarized fs-laser pulses can be varied between -40 to $+40 \mathrm{ps}$ with a temporal resolution of $\sim 0.1$ ps. The surface morphology of the irradiated areas was characterized by means of optical (OM), scanning electron (SEM) and scanning force microscopy (SFM).

For low fluences (where only the joint action of both laser pulses induces LIPSS) and temporally separated pulses, the polarization of the first laser pulse arriving on the surface determines the orientation of the LIPSS [1]. For temporally overlapping pulses, the coherent superposition of both polarizations determines the LIPSS orientation (Fig.1).

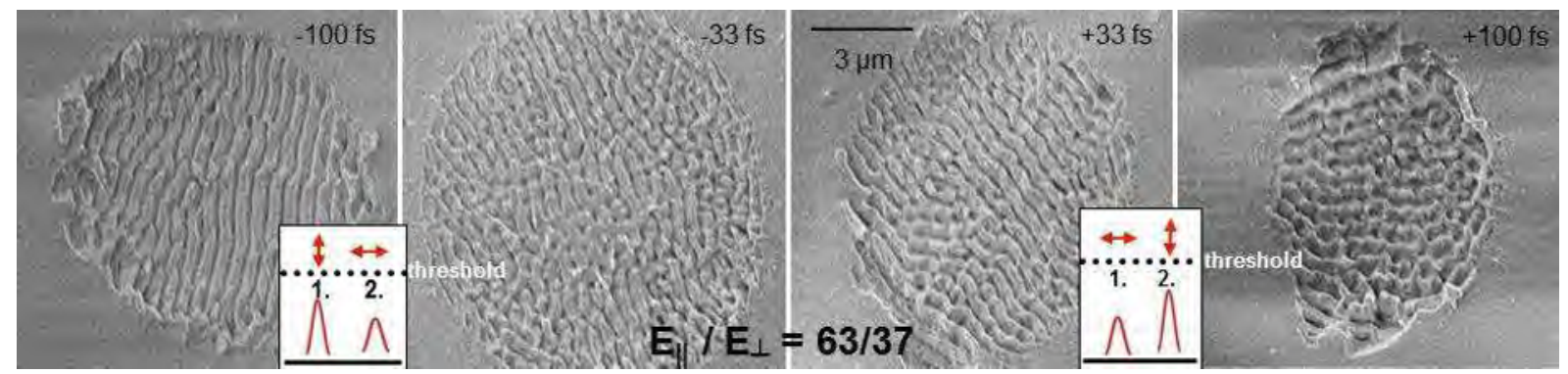

Fig. 1 SEM of a fused silica surface after the exposure to five double-50-fs-pulse sequences at a total peak fluence of 6.2 $\mathrm{J} / \mathrm{cm}^{2}$ for varying delays from $-0.1 \mathrm{ps}$ up to $+0.1 \mathrm{ps}$. The polarizations of the two pulses are perpendicular to each other. The insert visualizes the intensity of the double-pulse sequence in relation to the ablation threshold. Only both pulses together exceed the ablation threshold for five pulses. The energy ratio of the two pulses is $E_{\|} / E_{\perp}=63 / 37$. The polarization of the first pulse determines the LIPSS direction (parallel to its polarization) even if the pulse energy is less than that of the second pulse. For delays in the range of the pulse duration an intermediate rotation of the LIPSS direction is observed (see the two pictures in the middle).

Additionally pump-probe diffraction measurements were performed in transillumination geometry to study the early formation mechanisms of LIPSS. This approach reveals the generation dynamics of near-wavelength-sized LIPSS showing a transient diffraction at the corresponding spatial frequencies even before a permanent surface relief was observed by OM (Fig. 2).

The results confirm that the ultrafast energy deposition to the materials surface is essential and triggers subsequent physical mechanisms such as the formation of self-trapped excitons [2]. 


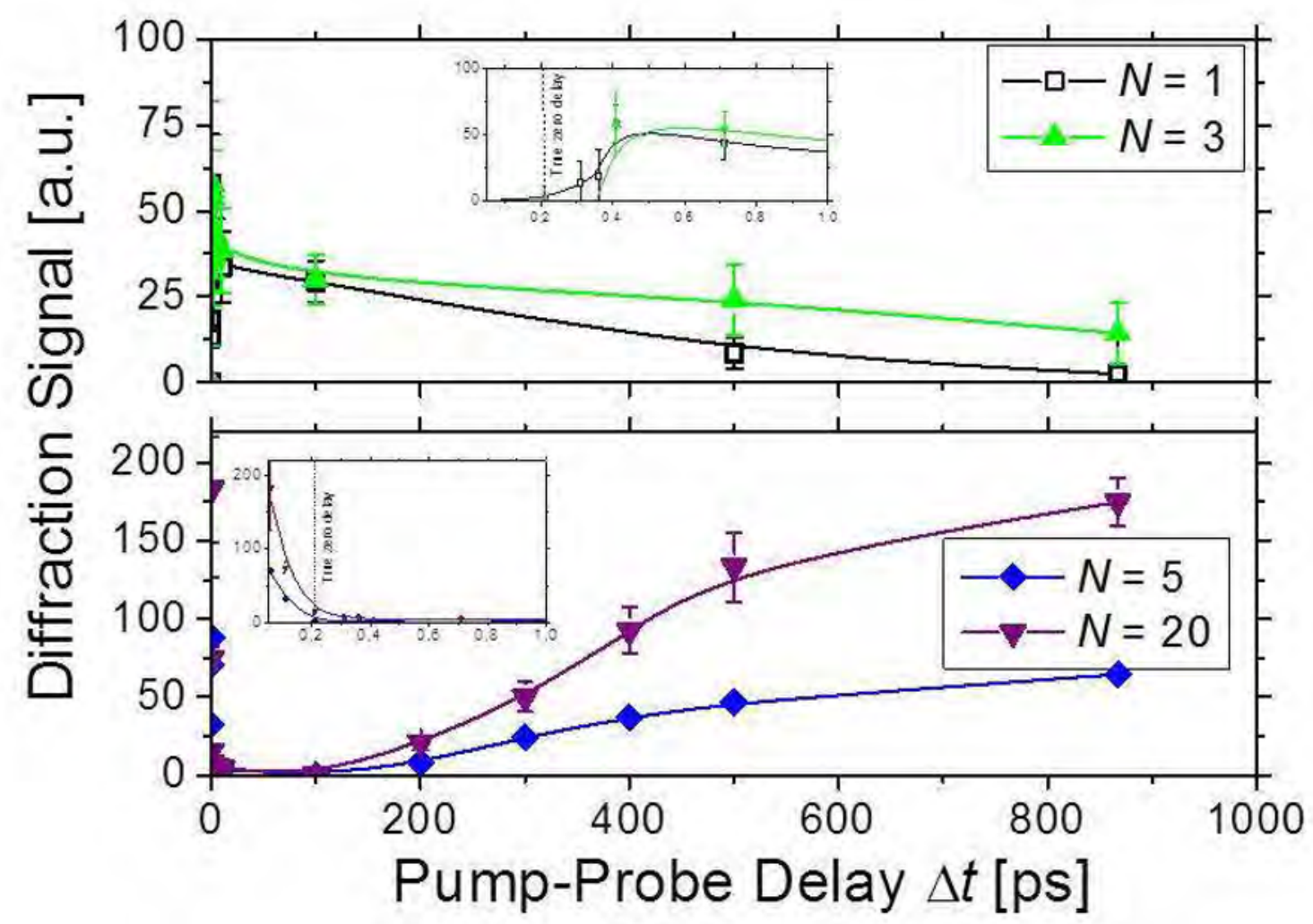

Fig. 2: Diffraction signal as a function of the pump-probe delay $\Delta t$ upon irradiation at $\phi_{0}=3.9 \mathrm{~J} / \mathrm{cm}^{2}$ by four different numbers of pump pulses $\left(\mathrm{N}_{\mathrm{Pump}}=1,3,5,20\right)$. The lines guide the eye. In the upper graph for these low pulse numbers no permanent ripple formation is observable but, a transient diffraction signal with a small rise time is seen. In the lower graph permanent ripple formation occurs and we observe a fast shielding of the optical signal by the arising of a strong electron plasma. The inserts indicate the short delay time behaviour of the corresponding diffraction signal.

\section{References}

[1] M. Rohloff, S. K. Das, S. Höhm, R. Grunwald, A. Rosenfeld, J. Krüger, and J. Bonse, Formation of laser-induced periodic surface structures on fused silica upon multiple cross-polarized double-femtosecond-laser-pulse irradiation sequences, J. Appl. Phys. 110, 014910 (2011)

[2] S. Höhm, A. Rosenfeld, J. Krüger, and J. Bonse, Femtosecond diffraction dynamics of laser-induced periodic surface structures on fused silica, Appl. Phys. Lett. (submitted) 